\title{
A channelling centre management software
}

\section{Dr. M.H.B. Ariyaratne}

Postgraduate Institute of Medicine, University of Colombo, Sri Lanka.

E-mail address: buddhika.ari@gmail.com

eHealth Sri Lanka 2010,1(suppl.1):S30

DOI: http://dx.doi.org/10.4038/sljbmi.v1i0.3589

Only the Abstract is available

\begin{abstract}
Most of the large scale medical channelling centres depend on a computer system these days. This presentation is on such a software system, currently implemented in two large private hospitals in the Southern Province and a few other medium scale channelling centres since more than four years. An unlimited number of consultants, categorised under their specialities, can be managed by this software. A single doctor can have multiple secessions for a day and each may have different attributes like the fee and starting time. The appointments are usually given as a serial number, but appointments can also be scheduled when necessary. The seessions are mainly changed by the day of the week.

During holidays the sessions can be given a different set of attributes like an earlier start and higher maximum number of patients. The doctors' leave allocations can also be managed by the system. Advance bookings, appointment cancellations, refunds, marking patients' pressence, reprints, name changes are all possible on the very same screen where bookings are done. Several reports like doctor view, nurse view, session view, shift end summary, day end summary are available and the frequently used ones are accessible through the main screen. The software is fully customisable with various printer settings, paper settings and printing positions. The appearance, the way of navigation, and several other features can also be customised. Virtually, an unlimited number of users can take appointments seamlessly in a network environment. The security is also a main concern and different authorities have various levels of accessibility.
\end{abstract}

Keywords - medical channelling centres, levels of accessibility, two large private hospitals, Southern Province 